\title{
IIEA
}
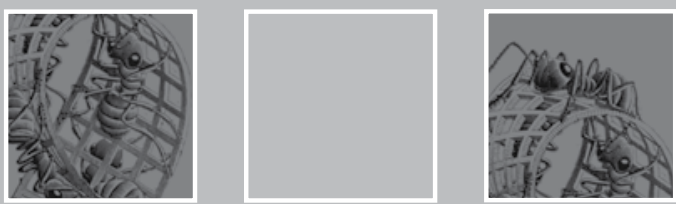

Tesis de

Maestría

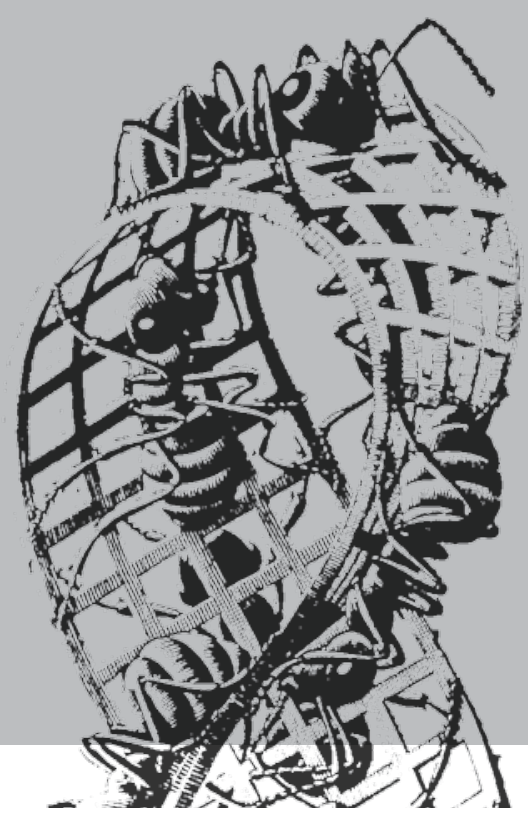




\title{
Maestría en Docencia de la Matemática
}

\section{Título: Acciones del profesor que promueven actividad demostrativa con estudiantes de sexto grado}

\author{
Yuri Tatiana Ospina Usaquén
}

Tania Julieth Plazas Merchán

Palabras clave: Actividad demostrativa, acciones del profesor, estudiantes de grado sexto, tareas.

\section{Descripción}

El interrogante que dio lugar a este trabajo era determinar si es posible propiciar actividad demostrativa con estudiantes de sexto grado, y qué acciones realiza la profesora para que ello ocurra. Para esto, se diseñaron y aplicaron tareas cuya intención era generar actividad demostrativa. Luego se identificaron las acciones de la actividad demostrativa que realizaron los estudiantes y se describieron las acciones del profesor durante el desarrollo de las tareas en clase; llamaremos clase B a las sesiones correspondientes en las cuales se propició la actividad demostrativa. Las acciones con las cuales se describió esta clase fueron seleccionadas de las propuestas por el grupo de investigación Aprendizaje y Enseñanza de la Geometría, $Æ \cdot G$, del Departamento de Matemáticas de la Universidad Pedagógica Nacional, de las cuales fue necesario modificar algunas $\mathrm{y}$ adicionar otras.

También se analizó una clase, que llamaremos clase A, desarrollada con el mismo grupo y la misma profesora, cuatro meses antes, en la cual no se tenía la intención de propiciar actividad demostrativa, para determinar cuáles de las acciones ya escogidas efectuaba la profesora. Dado el tipo de clase que se desarrolló, fue necesario incluir nuevas acciones. Finalmente, se realizó una comparación entre los análisis y descripciones de las dos clases, centrando la atención en las acciones de la profesora.

Este es un trabajo de grado de la Maestría en Docencia de las Matemáticas modalidad de profundización, asociada al grupo de investigación $Æ \cdot G$, quienes están interesados en identificar condiciones que favorezcan la actividad demostrativa en la secundaria.

\section{Contenidos}

Este documento consta de cinco capítulos organizados de la siguiente manera: en el capítulo uno se presentan los antecedentes para la realización de este documento, la formulación del problema, justificación y los objetivos generales y específicos. En el segundo capítulo se elabora el marco teórico que sustenta este trabajo. El capítulo tres corresponde a la metodología; allí se presentan las etapas en las que se realizó el proyecto que incluyen: la descripción 
de la clase $\mathrm{A}^{1}$, la planeación y desarrollo de la clase $\mathrm{B}^{2}$ y la determinación de las acciones del profesor para analizar la clase A y B. El cuarto capítulo muestra el análisis de las acciones del profesor en las dos clases. Por último, en el capítulo cinco se registran las conclusiones.

El documento tiene trece anexos: nueve presentan las actividades aplicadas en la clase B, en la cual se propició la actividad demostrativa, y una que presenta la actividad de la clase A, en la cual la intención no era que dicha actividad se diera. Hay dos trascripciones: una de la reunión de la profesora con la asesora y la otra de la clase A, y una tabla con el número de veces que se presentan las acciones en cada clase.

\section{Metodología}

El estudio llevado a cabo en este trabajo da cuenta de una investigación de tipo cualitativo descriptivo, ya que se recogió parte de los datos mediante registros audiovisuales de las clases para un posterior análisis, donde se establecieron y describieron las acciones del docente para favorecer la actividad demostrativa.

Por otro lado, la metodología denominada experimento de enseñanza sirve como referente metodológico para este trabajo de grado. Ello debido a tres aspectos: (1) tanto la postura de aprendizaje que subyace a la metodología como la perspectiva de aprendizaje sociocultural del constructo teórico actividad demos-

1 Clase en la cual no se tenía la intención de propiciar actividad demostrativa.

2 Clase en la cual se tenía la intención de propiciar actividad demostrativa. trativa coinciden. (2) Los objetivos de este trabajo están relacionados con dos de los asuntos que según Cobb (2000) permiten investigar experimentos de enseñanza: el aprendizaje matemático de los estudiantes en colaboración con los profesores y la actividad del profesor. (3) Las tres fases de un experimento de enseñanza, que según Cobb (2000), son el diseño instruccional y planificación, el análisis en curso de las actividades escolares y el análisis retrospectivo, tienen relación con las fases de desarrollo de este trabajo.

Además de identificar las acciones del profesor durante el desarrollo de una clase en la cual se quería propiciar la actividad demostrativa, se hizo lo mismo en una clase en la cual no se tenía esa intención, con el fin de establecer cuáles tienen incidencia en la posibilidad de desarrollar actividad demostrativa en el aula.

\section{Conclusiones}

Los resultados de este trabajo están basados en el análisis hecho a cuatro sesiones de clase desarrolladas por una profesora con un grupo de estudiantes de grado sexto; esto nos permite notar diferencias entre las acciones que un profesor realiza en el aula cuando su intención es propiciar actividad demostrativa y aquellas que hace cuando no es ese su propósito.

Una de las intenciones de este trabajo era determinar si es posible que estudiantes de secundaria hagan actividad demostrativa, específicamente estudiantes de grado sexto. Hemos evidenciado que ello es posible; sin embargo, debe modificarse el constructo de actividad 
demostrativa propuesto por el grupo de investigación $\nRightarrow$ · G.

El proceso de justificación de esta actividad está descrito como acciones que llevan a demostrar el hecho geométrico que formulan como conjetura en el proceso de conjeturación de dicha actividad. Dado que ello no era posible con las estudiantes de grado sexto, debido a que no contaban con la teoría que se necesitaba para demostrar la conjetura, ni experiencia en ese tipo de proceso, la modificación consistió en exigirles que justificaran otras tareas o situaciones haciendo uso del hecho geométrico descubierto.

Este cambio se dio por tres razones: la primera, como ya se mencionó, porque las estudiantes no estaban acostumbradas a este tipo de actividad; la segunda porque no hacía parte de las normas sociomatemáticas de clase argumentar sus procesos y afirmaciones; y la tercera porque no tenían un sistema teórico al cual recurrir para justificar los hechos geométricos descubiertos.

En general, las tareas propuestas influyen en gran parte la posibilidad de propiciar actividad demostrativa con estudiantes. Estas deben ser de tipo abierto, que pueden ser accesibles o difíciles, que le permitan al estudiante llevar a cabo exploraciones y visualizaciones con el uso de diferentes materiales, por ejemplo, palos de colores, uso de papel, regla y compás, o geometría dinámica, y que den lugar al descubrimiento de hechos geométricos, promuevan la generalización y exijan justificaciones. Sin embargo, el diseño y aplicación de estas tareas por sí solas no generan la actividad demostrativa. Para ello es necesario el propósito deliberado del profesor de favorecerla.

A partir del análisis de la clase A, concluimos que esta concuerda con lo descrito por Cantoral y Farfán (2003) como clase tradicional (ver capítulo 1), ya que en esta clase la profesora es la que dirige, presenta el concepto y propone ejercicios del libro, sin darle oportunidad a sus estudiantes de participar activamente en la construcción del conocimiento. Además, en las actividades propuestas por la profesora en la clase A, la demostración carece de sentido $\mathrm{y}$, por tanto, la actividad demostrativa también.

Como lo pudimos evidenciar en las acciones de la profesora, tanto en la clase A como en la clase B hay acciones que son usuales. Algunas de estas acciones tienen como fin que los estudiantes tengan claras las normas de la clase y el papel tanto del profesor como de los alumnos en el desarrollo de esta, para favorecer el aprendizaje. Ejemplos de estas son: dar verbalmente información relativa al funcionamiento de la clase (1), incentivar intervención de los estudiantes (14), proveer información (19) y dar información relativa al procedimiento $o$ la tarea (20). Otras acciones comunes están relacionadas con la reacción del profesor frente a las participaciones de sus estudiantes; por ejemplo, declarar, indicar, explicar o corregir el error (10), parafrasear aporte de estudiante (11) y aprobar el aporte del estudiante (12).

Cuando en la clase se desarrollaron las tareas diseñadas para propiciar actividad demostrativa, la profesora llevó a cabo más acciones de las que habitualmente hacía en clase, dado que esta actividad y las tareas propuestas 
demandan mayor autonomía y participación de los estudiantes, frente a lo cual el profesor debe intervenir y reaccionar de manera diferente a lo usual y hacer exigencias distintas a sus estudiantes. Las acciones relacionadas con lo anterior son: proporcionar espacio de reflexión (2), informarse sobre las acciones realizadas por los estudiantes al abordar una tarea durante el trabajo individual hecho en clase (3), informarse sobre los resultados geométricos obtenidos por los estudiantes durante el trabajo individual realizado en clase (4), aprovechar intervención de estudiante (7), concretar el resultado logrado hasta el momento (8), institucionalizar el saber (9), repreguntar (13), responsabilizar a los estudiantes del desarrollo de la tarea o de los conocimientos trabajados (15), exigir justificaciones (17) e indagar (18).

Además de favorecer el desarrollo de actividad demostrativa, la profesora tenía como intención mejorar el lenguaje matemático de sus estudiantes, lo cual implicó nuevas acciones en su clase, como por ejemplo reaccionar con aclaración o precisión (6) y exigir aclaración o precisión (16).

A continuación se presentan las acciones del profesor que se dieron con mayor frecuencia, durante el desarrollo de cada una de las acciones de la actividad demostrativa que se generaron en la clase B:

- Visualización y exploración: estas acciones se evidenciaron en el desarrollo del punto 1 de la actividad 3. Durante ese momento, la profesora se ve en la necesidad de informarse sobre las acciones que las estudiantes están realizando (4), para asegu- rarse de que ellas desarrollen el procedimiento correctamente y así que puedan lograr descubrir el hecho geométrico que subyace a la tarea.

- Generalización: para propiciar esta acción, es necesario que la profesora incentive a las estudiantes (14) constantemente, buscando que compartan sus descubrimientos individuales o grupales, para concretar los resultados (8) e institucionalizar el saber (9) ante todo el curso.

- Justificación: dado que las estudiantes deben utilizar el hecho geométrico descubierto para justificar la respuesta a una situación, las acciones de la profesora más comunes durante este proceso son: exige aclaración $o$ precisión (16), exige justificación (17), indaga (18) y provee o información (19).

Evidenciamos que las acciones del profesor mencionadas (acciones 4, 8, 14, $16,17,18,19)$, son propias y necesarias cuando se realiza una clase en la cual se tiene la intención de generar la actividad demostrativa. Esto por dos razones: la primera, porque no se presentan en la clase A, ya que son acciones que promueven la participación autónoma de los estudiantes, contrario a lo que ocurre en esta clase; y segundo, porque estas acciones, particularmente, impulsan a los estudiantes a realizar cada una de las acciones de la actividad demostrativa.

La participación de las estudiantes en los dos tipos de clase fue diferente, pues dado el tipo de tareas propuestas en la clase B, ellas fueron más autónomas y participativas. En este tipo de clase, 
el profesor no es el único responsable de generar conocimiento para que los estudiantes lo adquieran, sino que ellos deben participar en ese proceso, en este caso, descubriendo hechos geométricos por medio de las acciones de tipo heurístico de la actividad demostrativa. Por tanto, el profesor debe abrir el espacio para que el estudiante participe, es decir, dar la información pertinente y favorecer las intervenciones de los estudiantes, incentivándolos para que logren tener una participación real. Esto se puede evidenciar, por ejemplo, cuando las estudiantes socializan las conjeturas a las que llegaron en el segundo punto de la actividad 3 (ver en el capítulo 2: fragmento 5 y fragmento 9), conjeturas autónomas y que se dieron a partir de la confianza que adquirieran por trabajar en grupo y por la gestión de la profesora.

En la clase A la profesora no da oportunidad a las estudiantes para que participen en la construcción del conocimiento, sino que trata de dar toda la información necesaria.

Es de resaltar que, como se ve en las clases A y B, se implementaron diferentes metodologías en cada una, y que cambió el tipo de tareas propuestas, el papel del profesor y del estudiante, el material utilizado y hasta la forma de evaluar las participaciones de las estudiantes en la clase B. Las estudiantes de grado sexto, con las cuales se llevó a cabo el experimento, se adaptaron fácilmente al cambio metodológico. Ellas reconocieron los cambios presentados y les pareció más agradable la forma como se desarrolló la clase B. Esto lo pudimos evidenciar por medio de los comentarios que escribieron las alumnas en la tarea final del curso, que consistió en escribir un cuento sobre su apreciación de la clase de geometría. Algunas de las afirmaciones que hicieron en los cuentos son:

- Ana: “Todo ha cambiado en la clase, la organización, la metodología, las explicaciones de la profe, etc.... me gusta más ahora porque no es la típica clase..."

- Erika: “...profe es que la clase siempre es la misma eso me aburre un poco..." más adelante en su narración dice “...la profesora llevó algo diferente, todo el salón estaba feliz..."

- Paula: “... Ahora vemos los temas y los ponemos en práctica... y hacemos el trabajo en grupo..."

- Valentina: “Al principio la clase era solo ejercicios en el libro... las actividades se volvieron más recreativas..."

- Laura. "me gustan más las últimas clases porque hay más dinámica..."

- Luisa: “...llevó cámaras y guías entonces la clase fue más divertida..."

Este trabajo de grado nos permitió hacer una reflexión sobre nuestra práctica docente, al comparar la clase A con la clase $\mathrm{B}$, teniendo en cuenta que la clase A era el tipo de clase que hacíamos habitualmente. Notamos que podemos hacer otro tipo de clase, como la clase $\mathrm{B}$, que nos exige más como docentes en la planeación de la clase y en el rol que debemos desempeñar dentro del aula.

Sin embargo, nos da más satisfacción. Ello porque en este tipo de clases se le da la oportunidad a los estudiantes de participar activamente en la cons- 
trucción del conocimiento, lo cual hace más significativo su aprendizaje, dado que ellos mismos descubren los hechos geométricos y desarrollan mayor comprensión de estos. Sentimos que hemos contribuido más a su aprendizaje. En la clase A, el profesor se encarga de dar el conocimiento y los estudiantes se convierten en receptores de este, mientras que en la clase B ellos son constructores del conocimiento. Tanto el profesor como los estudiantes participan como miembros de una comunidad que tiene un mismo propósito.

\section{Título: Emergencia de procesos de la actividad demostrativa en una clase con estudiantes en edad extraescolar}

\section{Carolina María Luque Zabala \\ Luis Alejandro Robayo León}

Palabras clave: Actividad demostrativa, producción de teoremas, estudiantes en edad extraescolar, geometría dinámica.

\section{Descripción}

En este trabajo se presenta un estudio realizado con estudiantes en edad extraescolar, quienes estaban nivelando los grados octavo y noveno de educación media en el colegio Gabriel Echavarría, de Madrid (Cundinamarca), durante el segundo semestre del 2010. El propósito del estudio es indagar sobre la posibilidad de evidenciar el constructo de actividad demostrativa, propuesto por el grupo EםG en este contexto. El objetivo principal del estudio es identificar las acciones de los estudiantes en edad extraescolar que reflejan su involucramiento en los procesos de la actividad demostrativa en clase de geometría.

Para cumplir con el objetivo del estudio, en primer lugar se caracteriza lo que significa producir teoremas en la escuela, basándonos en el constructo de teorema propuesto por Mariotti (1997, 2006), y se diseñan categorías de análisis que permiten identificar si existe un involucramiento por parte de los estudiantes en la producción de teoremas y, por ende, en la actividad demostrativa. Estas categorías se basan en las fases de producción de teoremas propuestas por Boero (1999), a las que se les diseñan indicadores $\mathrm{y}$ acciones que permitan evidenciarlas.

En segundo lugar, hubo una fase de inducción que involucró a los estudiantes en una aproximación metodológica, en la que se estudia la geometría euclidiana a través de una participación activa, el trabajo en grupo y el uso de la geometría dinámica. Los análisis de la 
actividad de los estudiantes de acuerdo con dos situaciones problema donde una de ellas provee el hecho geométrico útil para justificar el establecido a partir de la otra.

Con base en el análisis, se elabora un reporte final en el que se concluye si existió un involucramiento en los procesos de la actividad demostrativa por parte de los estudiantes.

\section{Contenidos}

El presente trabajo se ha organizado en seis capítulos, distribuidos de la siguiente manera: en el primero, se describe la problemática, se presentan los objetivos y la revisión de antecedentes de acuerdo a los intereses del estudio. En el segundo se presenta el referente teórico que fundamentó el estudio. En el tercer capítulo se describe el diseño metodológico del estudio, centrado en tres aspectos: el tipo de investigación, las características de la población cuyas acciones fueron objeto de estudio y las etapas que lo conforman. El cuarto trata sobre las categorías de análisis utilizadas para analizar la actividad de los estudiantes. En el capítulo cinco se expone el análisis y los resultados de la investigación. En el capítulo seis se mencionan las conclusiones del estudio en relación con la actividad de los estudiantes, los objetivos del estudio, los aspectos a tener en cuenta en futuros estudios y las reflexiones sobre la incidencia del trabajo realizado en nuestra formación personal y profesional.

\section{Metodología}

De acuerdo con los intereses del estudio, la investigación se enmarca en una metodología de carácter cualitativo, descriptivo e interpretativo, que corresponde aun estudio de caso. Es un estudio de caso porque se busca describir las acciones que realiza un grupo de tres estudiantes, cuando se enfrenta a una situación geométrica que indaga sobre las propiedades del triángulo. Para ello, se realizaron unos primeros registros de audio y video de las sesiones trabajadas, entrevistas y se usaron las producciones escritas de los estudiantes. Posteriormente, se hizo la transcripción de las sesiones en las que los estudiantes solucionaban dos situaciones problema que involucraban la conjetura de un hecho geométrico. Paralelo al registro de estos datos, se diseñaron las categorías de análisis con las cuales se analizarían las transcripciones. El establecimiento de las categorías de análisis nos permitió seleccionar y depurar los episodios que finalmente se considerarían los datos del estudio.

\section{Conclusiones}

En relación con la actividad de los estudiantes en edad extraescolar, se evidenció que las acciones de la actividad demostrativa emergen en una clase en la que la geometría dinámica, la interacción social y la solución a situaciones problema se articulan para conducir a los estudiantes a producir conjeturas y justificaciones desde el punto de vista de la actividad demostrativa.

La actividad de los estudiantes en edad extraescolar permitió reconocer la necesidad de ampliar el constructo de actividad demostrativa para el contexto escolar, como por ejemplo, extender las definiciones que se dan a las acciones de verificar y explorar. En relación con la exploración, los estudiantes dieron 
muestra de diferentes maneras de explorar: arrastrar para identificar invariantes, tomar medidas para identificar relaciones entre los objetos involucrados en la situación y lanzar hipótesis de solución, para luego corroborarlas haciendo uso de las herramientas de Cabri. Por otra parte, la acción de verificación, a diferencia de la definida en el constructo de actividad demostrativa, no solo se lleva a cabo cuando los estudiantes van comprobar un invariante encontrado, también cuando verifican una hipótesis de solución y construcciones realizadas.

Otro aporte de este trabajo en relación con el aprendizaje de la demostración en la escuela es la concepción de teorema que proponemos para al contexto escolar. En este sentido, planteamos una analogía entre los elementos que componen la definición de teorema propuesta por Mariotti (1997) y los elementos que están inmersos en el constructo de actividad demostrativa y que consideramos son próximos a tal definición en el contexto escolar.

Por otro lado, el análisis de la actividad de los estudiantes en edad extraescolar nos ha llevado a considerar cuestiones para futuros estudios. Algunas de ellas son: ¿en qué sentido sería provechoso, o no, proponer situaciones próximas al contexto laboral de los estudiantes para la construcción del conocimiento y la emergencia de acciones de la actividad demostrativa? Teniendo en cuenta que los estudiantes lograron plantear justificaciones cuando apenas se estaban iniciando en el proceso de justificar, ¿de qué manera se orientaría el trabajo con los estudiantes para dar continuidad a la producción de justificaciones próximas a una demostración formal?

\section{Título: Acciones del profesor que favorecen el desarrollo de la actividad demostrativa en grado noveno}

Ivon Pinzón

Antonio Rodríguez

Palabras clave: Actividad demostrativa, geometría, acciones del profesor, geometría dinámica.

\section{Descripción}

El presente trabajo de grado es el resultado de un conjunto de acciones investigativas llevadas a cabo para optar por el título de Maestría en Docencia de la Matemática. La propuesta se ubica dentro de la línea de investigación Aprendizaje y Enseñanza de la Geometría $(Æ \cdot G)$ de la Universidad Pedagógica
Nacional, que se ha centrado en el estudio de elementos que intervienen en la enseñanza y el aprendizaje de la demostración en Geometría, teniendo como trasfondo un programa de formación de docentes. El trabajo consiste principalmente en la descripción y análisis de las acciones que lleva a cabo el profesor en 
el aula con estudiantes de grado noveno para promover el desarrollo de actividad demostrativa.

\section{Contenidos}

El documento está organizado en cuatro capítulos: marco teórico, acciones investigativas, análisis de la información y conclusiones.

En el marco teórico se tratan tres elementos. Primero, el constructo actividad demostrativa, reconocido como vía para el aprendizaje de la Geometría y conformado por los procesos de conjeturación y justificación. Segundo, las acciones del profesor, que se consolidan como un elemento importante para propiciar la actividad demostrativa. Tercero, las herramientas de mediación en el aula, que contribuyen al descubrimiento de propiedades y relaciones entre objetos geométricos; en particular hacemos referencia al software de geometría dinámica Cabri. Sobre las acciones investigativas y análisis de la información haremos énfasis en el siguiente apartado.

\section{Metodología}

Es necesario señalar que diseñamos un conjunto de actividades con las que se esperaba involucrar a los estudiantes en un proceso de aprendizaje del uso de geometría dinámica, y a través de ello, dar lugar al desarrollo de las acciones de la actividad demostrativa. Sin embargo, debido a las discusiones dadas en los espacios de asesoría, surgió la necesidad de crear un guión del profesor, con el fin de dirigir, de manera puntual, su actuación frente a las acciones de los estudiantes en el desarrollo de las tareas.

En cuanto al desarrollo del análisis, se llevó a cabo atendiendo a tres momen- tos: análisis de las tareas propuestas, codificación de las acciones del profesor e identificación de acciones, codificación y análisis. En el primer momento hicimos el análisis de las diferentes tareas diseñadas en relación con el favorecimiento de acciones propias de la actividad demostrativa. En el segundo momento, se establecieron y codificaron aquellas acciones del profesor, propuestas en la teoría, que considerábamos pertinentes para el análisis de los acontecimientos en el aula. En el tercer momento, se codificaron las intervenciones de los estudiantes relacionándolas con las acciones de la actividad demostrativa y se identificaron las acciones del profesor que las originaron.

\section{Conclusiones}

El planteamiento de las conclusiones de este trabajo se da con base en las ideas que se describen a continuación:

La actividad demostrativa es un constructo que se puede desarrollar en el aula de clase con estudiantes de educación básica. Además, no es suficiente identificar las acciones de la actividad demostrativa en el trabajo de los estudiantes sino que es necesario también establecer indicadores de ellas.

Para realizar un estudio como el que aquí se reporta, se evidencia la importancia de utilizar mecanismos de registro de información como audio y video.

Las acciones del profesor propuestas por el grupo de investigación $Æ \cdot G$ de la Universidad Pedagógica Nacional, se presentan como un elemento suficiente para este tipo de análisis.

Dados los resultados del trabajo, identificamos la importancia de buscar 
condiciones que permitan llevar a cabo este tipo de experiencias con todos los estudiantes de secundaria.

$\mathrm{Al}$ estudiar las acciones del profesor para propiciar la actividad demostrativa de sus estudiantes, se presentó también la necesidad de realizar un diseño de tareas adecuado, siendo este último un aporte más a nuestro trabajo.

El profesor que lideró el desarrollo de las actividades en el aula, y que es uno de los autores de este trabajo, alcanzó a percibir una gran diferencia en el ambiente de clase durante el desarrollo de estas actividades, a diferencia de cuando usaba la metodología tradicional de enseñanza.
Reconocemos la pertinencia de llevar a cabo un posterior trabajo de investigación en el que se involucre toda una clase, para determinar si los resultados obtenidos son similares.

Destacamos que a pesar de que el constructo actividad demostrativa fue diseñado pensando en estudiantes universitarios, es posible que los estudiantes de secundaria realicen la actividad demostrativa en toda su dimensión, como fue concebida, siempre y cuando cuenten con un sistema teórico para la Geometría en el cual apoyarse para construir una justificación. 


\title{
Maestría en Docencia de la Química
}

\author{
Título: Del concepto tetravalencia a la tridimensionalidad \\ del átomo de carbono: aportes desde los estudios \\ histórico-epistemológicos a la construcción de una ruta \\ didáctica
}

\section{Elizabeth Casallas Rodríguez}

Palabras clave: Historia y epistemología, unidad didáctica, modelos, enseñanza de la química, experimentación, tetravalencia, tridimensionalidad.

\section{Descripción}

El presente trabajo de investigación, muestra la ruta y la propuesta de intervención didáctica que se derivaron de una mirada al contexto históricoepistemológico en que surge el concepto de tetravalencia del átomo de carbono; de la revisión de 8 libros de texto (media vocacional y universitario) para la enseñanza de la química en la educación media vocacional, para lo cual se emplearon seis criterios de análisis que surgieron de la revisión de documentos originales y fuentes de segundo orden; y de un acercamiento a la situación actual de la enseñanza de la química en la educación media.

\section{Contenidos}

El presente trabajo está organizado en siete capítulos centrales, además de los correspondientes a conclusiones, recomendaciones, bibliografía y anexos.

En el primer capítulo, se hace la presentación del proyecto en general, donde se incluyen los objetivos, justificación, planteamiento del problema y metodología a seguir. El segundo capítulo corresponde a la mirada que se hace al contexto histórico epistemológico en que surge el concepto de tetravalencia del átomo de carbono; allí se describen las principales características de la afinidad química, se revisan las teorías que eran vigentes en la primera mitad del siglo XIX, se abordan las alianzas que surgen entre la química mineral y la orgánica y, finalmente, se analizan aspectos relevantes, relacionados con la tetravalencia del átomo de carbono, como la isomería.

En el tercer capítulo se abordan elementos relacionados con la situación actual (una aproximación) de la enseñanza de la química en la educación media vocacional, desde dos ámbitos: el primero, hace referencia a algunas investigaciones relacionadas con la enseñanza de la tetravalencia y de la tridimensionalidad del átomo de carbono, o con conceptos que guardan gran relación con estos; el segundo, corresponde a investigaciones relacionadas con las prácticas pedagógicas, particularmente de la enseñanza de la química; el tercero hace énfasis en el papel de la 
experimentación en la enseñanza de la química; el cuarto ámbito, está dedicado a analizar la importancia de los modelos en la actividad pedagógica de la química; el quinto, hace referencia a los libros de texto que se emplean en la enseñanza de la química; y finalmente, el sexto, en el que se presentan los resultados obtenidos en el análisis de los 8 textos más empleados en la enseñanza de la química (5 de ellos corresponden a los utilizados por los profesores de la institución educativa donde se desarrolló la presente investigación).

El cuarto capítulo está dedicado al estudio de caso. En este se describen sus principales características, los elementos que tiene en cuenta para su formulación y desarrollo. Por otra parte, también se incluyen elementos de la ruta de intervención didáctica, en la que se describen las cuatro etapas que la conforman, indicando las intenciones y las actividades que se proponen para cada una; de igual forma, se incluye la síntesis que se hace de las explicaciones y representaciones de los estudiantes, a partir del análisis de cada uno de los registros obtenidos en las diferentes actividades.

El capítulo cinco está dedicado a presentar las conclusiones que se derivaron de los objetivos específicos, de la pregunta de investigación y de las explicaciones y representaciones que hacen los estudiantes a partir del comportamiento de las sustancias con las que se vinculan a diario.

En el capítulo seis se presentan las recomendaciones que se derivan de todo el trabajo realizado, es decir, del diseño, implementación y análisis de los resultados obtenidos, las cuales serán tenidas en cuenta tanto por la autora, como por la directora para futuras investigaciones en este campo. El capítulo siete corresponde a las referencias bibliográficas, que proporcionaron las bases teóricas, epistemológicas y didácticas que soportan el presente proyecto de investigación.

En el capítulo octavo se presentan los anexos, que corresponden a: instrumentos como la encuesta aplicada a los docentes de las instituciones educativas, que participaron en la selección de los libros de texto a los que se le realizó el análisis de texto; las encuestas aplicadas a los docentes de la I.E.D. El Minuto de Buenos Aires, con el propósito de determinar las metodologías empleadas en la enseñanza del concepto tetravalencia, y los resultados obtenidos por los estudiantes, en términos aprendizaje del concepto tetravalencia y de la representación que hacen de la tridimensionalidad del átomo de carbono; también, en este capítulo se presenta la unidad didáctica.

\section{Metodología}

Se desarrolló una metodología cualitativa, mediante el diseño de estudio de caso único de una intervención didáctica desarrollada en la Institución Educativa Distrital El Minuto de Buenos Aires, con 33 estudiantes que cursan grado once (educación media vocacional).

Se hace un acercamiento al contexto histórico epistemológico en que surgió el concepto tetravalencia del átomo de carbono, el cual deriva seis criterios que fueron empleados en la revisión de los cinco textos más empleados para la enseñanza de la química en esta institución educativa, y tres libros de carácter universitario que tanto la autora como 
otros docentes de la Universidad Manuela Beltrán y de la Universidad Distrital, han utilizado a lo largo de su experiencia docente. De otra parte, también se tuvo en cuenta la aproximación que se hace a la situación actual de la enseñanza de la química en la educación media.

Todos estos elementos derivaron en una serie de criterios epistemológicos, cognitivos y pedagógicos que se pusieron en juego en la ruta didáctica y luego en el diseño de la unidad didáctica que se aplicó a los estudiantes de la institución educativa mencionada anteriormente, para favorecer la comprensión del concepto tetravalencia del átomo de carbono y de la representación que hacen de la tridimensionalidad de las moléculas.

\section{Conclusiones}

El acercamiento al contexto histórico en el que se desarrolla el concepto tetravalencia, permitió identificar los problemas más relevantes que se presentaban en la época, algunos de ellos son:

- Las teorías que de cierta forma eran rivales (de los radicales y la de los tipos).

- La preocupación por clasificar la gran cantidad de sustancias con las que se contaba hasta el momento.

- La determinación de la nomenclatura, que se emplearía precisamente para clasificar las sustancias existentes hasta entonces.

- Determinar la organización y comportamiento de los átomos dentro de las sustancias (moléculas).
Las controversias y rivalidades presentadas entre los diferentes científicos (determinadas en cierta forma por el poder con el que contaban). Entre algunos de ellos podemos citar: (a) Laurent y Berzelius: el primero criticó los trabajos realizados por Berzeluis, donde propone que sustancias como el ácido tricloroacético están formados por compuestos copulados (Partington, 1965, p. 259); (b) Dumas y Berzelius: cuando este último acusa a Dumas de haber emitido la "teoría absurda" de que el cloro desempeña el mismo papel que el hidrógeno en los derivados de sustitución, a lo cual Dumas responde: "No soy responsable de la generalización exagerada que Laurent ha hecho de mi teoría". Sin embargo, cuando descubrió el ácido tricloroacético, Dumas contempló la posibilidad del éxito de la teoría y reclamó para él la exclusividad de la misma; (c) las disputas entre Dumas y Liebig ocasionadas por la naturaleza del radical benzoilo y por la composición de los éteres: en esta pelea, Berzelius ejerce como mediador, pero luego se une con Liebig en contra de Dumas, cuando este último publica su estudio sobre el ácido cloroacético, donde Berzelius defiende su teoría de radicales. Por su parte, Liebig denuncia la arrogancia de Dumas y sus aliados y jura censurar sus trabajos en su revista (Bensaude y Stengers, 1997, p. 112); (d) la disputa que se generó entre Couper y Kekulé, ocasionada por la autoría del desarrollo de la idea de la tetravalencia del carbono y de su cadena, ya que ellos dos la hicieron por separado, pero como Couper no era miembro de la Academia de Ciencias Francesa, la presentación de su trabajo fue aplazada hasta después 
de la publicación del artículo de Kekulé en los Annalen en 1858. (Brock, 1992, p. 226).

Algunos de los elementos mencionados anteriormente fueron base para el desarrollo de la propuesta en este trabajo de investigación.

El análisis de contenido al que fueron sometidos los libros de texto seleccionados para tal fin, permitió determinar que presentan el concepto tetravalencia como algo terminado, definitivo y despojado de su contexto históricoepistemológico. Por otra parte, no abordan los problemas que motivaron su surgimiento, como tampoco hacen alusión a las prácticas experimentales que fueron realizadas y que permitieron la construcción de este concepto; situación que no solo se presenta con este concepto, sino que se ha encontrado, en otras investigaciones, que la ciencia en general se presenta como una actividad simplificada, individual, absolutista y reduccionista, totalmente alejada de una realidad, en cuanto a que no hay un acercamiento al contexto histórico en el que son presentadas las propuestas de los científicos (Cuellar, Pérez y Quintanilla, 2005, p. 2).

El acercamiento a la situación actual de la enseñanza de la química permitió derivar implicaciones pedagógicas, principalmente en la necesidad de replantear el currículo de química en la I.E.D. El Minuto de Buenos Aires, teniendo en cuenta los siguientes aspectos: acercamiento al contexto histórico-epistemológico en que surgen los conceptos químicos, concretamente el de tetravalencia; planteamiento de problemas que vinculen el comportamiento químico de las sustancias con unas formas de representación (modelos, tanto teóricos como didácticos construidos por ellos); una metodología que involucre la realización de prácticas experimentales (en lo posible con sustancias de uso cotidiano), como herramientas que promuevan en los estudiantes, entre otras cosas, una actitud científica y el aprendizaje del concepto tetravalencia del átomo de carbono.

\section{Título: Aplicación de conceptos y relaciones estequiométricas en el trabajo práctico experimental}

\section{Nubia Liliana Ramírez Ospina}

Palabras clave: Trabajo práctico experimental, enseñanza, aprendizaje significativo, estequiometría, diagramas heurísticos.

\section{Descripción}

Esta investigación consiste en el diseño e implementación de una estrategia didáctica fundamentada en el trabajo práctico experimental para la enseñanza y el aprendizaje de la estequiometría, sus conceptos y las relaciones que se dan en esta rama auxiliar de la Química. La estrategia metodológica está construida 
en el modelo de los trabajos prácticos experimentales de Aurelí Caamaño. La evaluación del aprendizaje significativo se hizo desde aspectos cualitativos tales como la observación, comparación, el manejo de mapas conceptuales, el desarrollo de diagramas heurísticos (Chamizo, 2009), la resolución de problemas y la implementación de analogías para ello. Desde el aspecto cuantitativo, se hizo un análisis estadístico de los resultados obtenidos en la implementación de los instrumentos planteados.

En el desarrollo de la investigación se identifica en los estudiantes un nivel inicial o novato en el manejo de conceptos relacionados con el cambio químico, leyes ponderales de la química y los aspectos cuantitativos que se generan desde la relación entre estos conceptos, así como la dificultad para proponer estrategias experimentales que les permitieran comprobar fenómenos. Posteriormente y con el desarrollo de trabajos prácticos experimentales, los estudiantes se fueron familiarizando con el proceso de construcción o diseño de posibles experimentos que les acerquen a la interpretación de fenómenos y por ende facilitar el aprendizaje mediante la ilustración de conceptos y la interpretación de experiencias (Caamaño, 2006).

\section{Contenido}

A continuación se muestran los referentes sobre los cuales se fundamenta la investigación:

Referente disciplinar: en este apartado se resume qué es la estequiometría, quién la postuló, en qué se fundamenta y cuáles son los conceptos primordiales, tales como leyes ponderales de la química, reacción química, mol, número de
Avogadro, para comprender esta rama auxiliar de la Química. De igual forma, se incluye la aplicabilidad de la estequiometría, la cual fundamentará los núcleos de la unidad didáctica.

Referente didáctico y pedagógico: en esta sección se describe brevemente el fundamento de los implementos que permiten desarrollar la investigación pedagógica, tales como el enfoque en resolución de problemas, el trabajo práctico experimental y las analogías como apoyo para la enseñanza de la química.

\section{Metodología}

La investigación comprende cuatro fases:

Fase I: diseño de instrumentos; planteamiento de un instrumento de ideas previas y construcción de una unidad didáctica.

Fase II: diagnóstico; el instrumento individual de ideas previas busca establecer el nivel en que se encuentra cada estudiante, de acuerdo con cada uno de los tipos de aprendizaje significativos.

Fase III: implementación de la unidad didáctica; la unidad se estructuró en cuatro núcleos que permitieron desarrollar las temáticas relacionadas a la estequiometría; de igual manera, están soportadas en el trabajo práctico experimental en contexto y presenta diversas actividades que sirven de analogía para entender la química.

Fase IV: seguimiento y evaluación de aprendizajes; esta fase está presente en toda la investigación e incluye una evaluación generada por los estudiantes quienes de manera anónima expresan sus opiniones sobre la unidad. 
Posteriormente a estas fases, se realizó el análisis de los resultados obtenidos a partir de las actividades planteadas en la unidad didáctica.

\section{Conclusiones}

Sobre el diagnóstico: el reconocimiento de las ideas previas que tienen los estudiantes, permitió identificar las temáticas que debían ser reforzadas mediante el desarrollo de la unidad didáctica. Se aprecia que en el inicio de la investigación, el nivel que presentan los estudiantes con respecto a los conceptos fundamentales de la química para enten- der la estequiometría, es inicial o novato: tan solo algunos estudiantes alcanzan el nivel intermedio o amateur.

Sobre la unidad didáctica: teniendo en cuenta que la unidad está estructurada desde el trabajo practico experimental, estas permitirían que los estudiantes aprendieran de forma ilustrativa las aplicaciones de la estequiometría.

Relacionar analogías con la resolución de problemas, es una manera efectiva para acercar la química a un lenguaje más escolar y cotidiano, permitiendo un mayor acceso de los estudiantes hacia los conceptos químicos.

\title{
Título: Competencia interpretativa y habilidades de pensamiento
}

\author{
Dora Ocampo Rozo \\ Dein Jeinman Cruz Leiva
}

Palabras clave: Competencia interpretativa, habilidades básicas de pensamiento, modelos didáctico-metodológicos COL, niveles de comprensión, bitácora COL.

\section{Descripción}

En esta investigación se realiza un análisis cuantitativo y cualitativo sobre la influencia ejercida por un programa de intervención cognitiva, basado en el Modelo de Comprensión Ordenada del Lenguaje (COL). A partir de la aplicación de este modelo se busca desarrollar la competencia interpretativa, a través del ejercicio de las habilidades básicas del pensamiento, en alumnos de grado décimo de la I.E San Antonio del municipio de Garzón (Huila), abordando las temáticas correspondientes al área de Química.

\section{Contenidos}

La investigación estuvo enmarcada en la caracterización inicial de las habilidades de pensamiento básicas para la adquisición y desarrollo de la competencia interpretativa (observación, relación y comparación) (Campirán, 1999), a través de la aplicación de un programa de intervención cognitiva y la evaluación del impacto del programa mediante la comparación entre los resultados de pruebas pretest y postest. 


\section{Metodología}

La metodología que se lleva a cabo es cuantitativa dentro del paradigma empírico-analítico, con un diseño de muestras cronológicas del tipo $\mathrm{O}_{1} \mathrm{XO}_{2} \mathrm{O}_{3}$ $\mathrm{XO}_{4} \mathrm{O}_{5} \mathrm{XO}_{6}$ sin grupo control, en el que $\mathrm{O} 1, \mathrm{O} 3 \mathrm{y} \mathrm{O}_{5}$, corresponden a las pruebas pretest y $\mathrm{O}_{2}, \mathrm{O}_{4}$ y $\mathrm{O}_{6}$ corresponden a las pruebas postest; $\mathrm{X}$ corresponde a las tres intervenciones, las cuales estructuran el programa de intervención cognitiva basado en el modelo de COL. La muestra fue intencional, ya que los sujetos no son asignados al azar, estos grupos ya estaban formados antes de la intervención. La población objeto de estudio consta de 21 estudiantes de décimo grado, entre los 15 y 17 años de edad de la I. E. San Antonio, de Garzón (Huila).

\section{Conclusiones}

La intervención didáctica y metodológica basada en el modelo de COL, permite un desarrollo en la competencia interpretativa mediante la ejercitación de las HBP que fueron evaluadas durante esta investigación, lo cual se puede evidenciar mediante el aumento de los niveles de asertividad de los estudiantes, obtenidos mediante la aplicación de las pruebas pretest y postest.

A través de este programa de intervención cognitiva, basado en el modelo de COL, se logró la ejercitación de las habilidades básicas de observación, comparación y relación, en donde se obtuvo un avance significativo en las habilidades de comparación y relación, evaluadas mediante las pruebas aplicadas. Sin embargo, en la habilidad de observación, los resultados no mostraron un avance progresivo durante el transcurso de las intervenciones, posiblemente porque el estudiante asume que la observación es una de las habilidades más sencillas y por tal motivo no lleva a cabo el proceso de forma detallada y minuciosa, restándole importancia y dedicación al proceso interpretativo de las preguntas de observación.

La complejidad de los temas es una variable muy importante, que se debe tener en cuenta en el momento de medir el progreso en las HBP durante el transcurso de las intervenciones. A medida que se avanzaba en ellas, durante esta investigación, aumentaba el nivel de complejidad de los temas, por tal motivo los resultados eran muy variados. Otro motivo que se debe tener en cuenta en la variabilidad de los resultados de las HBP, es la inasistencia de los estudiantes en el desarrollo de las actividades propuestas, lo cual impedía una normal secuencia en el desarrollo de las HBP evaluadas.

El progreso en la competencia interpretativa, y por consiguiente de cada una de las HBP evaluadas, está determinado también por el nivel de conocimientos que posea un estudiante sobre los temas tratados. En la primera intervención, referente a la materia, los temas estudiados en ella son bastante trabajados en años anteriores; por tal motivo, no se notó un progreso significativo entre el pretest 1 y el postest 1 , dado que los resultados en pretest fueron buenos. Sin embargo, los resultados en los niveles de asertividad y los obtenidos en la medición de la eficiencia del programa de intervención permiten afirmar un 
significativo avance en el desarrollo de la competencia interpretativa.

El modelo de COL, es una propuesta que posee los componentes básicos y necesarios para la estructuración de un programa de intervención metodológica y didáctica. El submodelo de orden de pensamiento y su estructuración contiene algunos elementos básicos de una metodología por investigación, tales como el planteamiento de una problemática, su hipótesis y trasfondo. 


\title{
Maestría en Tecnologías de la Información aplicadas a la Educación
}

\author{
Título: Escenario de interacción de socializaciones en \\ ambientes e-learning para la asimilación de conceptos \\ sobre el Concejo de Bogotá
}

\section{Giovanni Guatibonza}

Palabras clave: Socialización, interacción, clases sociales, aprendizaje colaborativo, representación del conocimiento, asimilación de conceptos.

\section{Descripción}

El presente trabajo de grado y proyecto pedagógico, es la implementación de un aula virtual de interés para estudiantes de educación media, con la finalidad de construir un escenario virtual en que personas provenientes de distintos orígenes socioeconómicos pudieran interactuar y consolidar conocimientos de manera horizontal. La interacción, además, permite identificar aquellas diferencias frente a la asimilación de conceptos de aquellos estudiantes que interactúan con personas de su mismo origen socioeconómico, frente a los que lo hacen con personas de distinto origen o escenario de socialización.

\section{Contenidos}

Con el ánimo de dar cuenta del objetivo principal del proyecto (determinar las diferencias en la asimilación de conceptos entre dos grupos de estudiantes que interactúan en un ambiente e-learning basado en el trabajo colaborativo, cuando un grupo proviene de distintos espacios de socialización y otro de un único espacio de socialización), el documento presenta una reflexión sobre los aspectos preliminares que llevaron a la elaboración de esta investigación: la justificación, el planteamiento del problema y los objetivos propuestos; los antecedentes desde las áreas teóricas planteadas; los principales acercamientos teóricos; los lineamientos metodológicos utilizados; el diseño y construcción del ambiente de aprendizaje; el análisis de la información; las conclusiones; por último, proyecciones y pasos a seguir.

\section{Metodología}

Siguiendo los lineamientos de la metodología GRACE, se elaboró un modelo de enseñanza denominado modelo de conexión de socializaciones. Igualmente, se diseñó un aula virtual en plataforma Moodle y se determinó como dominio de conocimiento el Concejo de Bogotá. El proyecto fue implementado a estudiantes de educación media y se implementó en los colegios 21 Ángeles 
de la localidad de Suba y Gimnasio Moderno de la localidad de Chapinero, reconociendo que los estudiantes de las dos instituciones obedecen a orígenes socioeconómicos distintos.

\section{Conclusiones}

Se determina que no hay una diferencia en la asimilación de conceptos entre estudiantes provenientes de escenarios de socialización distintos. No obstante, se determina que aquellos estudiantes que obtuvieron diferencias más amplias entre la evaluación comparativa de sus mapas conceptuales iniciales y finales, son aquellos que no tuvieron interacción de socializaciones. Los resultados obtenidos se encuentran impactados por inconvenientes referentes a la implementación, esto debido a que se debió reducir el número de semanas de actividades, como también a que el desarrollo de trabajo, principalmente en sesiones presenciales, dificultó la ampliación de la formación propuesta en la virtualidad.

Se reconoce el trabajo de un grupo de interacción de socializaciones con un buen rendimiento en su evaluación, como el punto de partida para mejorar el modelo de enseñanza, formular nuevos dominios de conocimiento y determinar nuevos modelos de evaluación, con el ánimo de implementar nuevamente este proceso y con ello potenciar el avance de escenarios académicos de interacción de socializaciones.

\section{Título: Habilidades del siglo XXI: evidencias de pensamiento sistémico, razonamiento deductivo y trabajo colaborativo en la solución de problemas a partir de un ambiente e-learning basado en un juego tipo puzzle}

\section{Jorge Mario Karam Rozo}

Camilo Alexander Mendieta Ortiz

Oscar Yecid Ramírez Cano

Palabras clave: Habilidades del siglo XXI, pensamiento sistémico, trabajo colaborativo, razonamiento deductivo, solución de problemas, videojuegos, juegos serios, puzzle, aprendizaje e-learning.

\section{Descripción}

Investigación cuasi experimental que correlaciona la evidencia de habilidades de pensamiento sistémico, razonamiento deductivo y trabajo colaborativo, con la eficiencia en la solución de problemas formulados a través de ambiente virtual de aprendizaje AVA con objetos virtuales de aprendizaje OVAS basados en juegos tipo puzzle.

Los juegos se articularon a través de una historia que lograra captar la atención de los usuarios y permitiera 
exponer la presencia de las habilidades objeto de la investigación.

Se trabajó con una población universitaria que cursa pregrado en enfermería, con edad promedio de 18 años.

\section{Contenidos}

El primer objetivo de la presente investigación fue contribuir al estudio de las HSXXI aplicadas en el contexto de la solución de problemas.

Se buscó evidenciar la presencia de habilidades de pensamiento sistémico, razonamiento deductivo y trabajo colaborativo en la interacción con videojuegos tipo puzzle, con el apoyo de un ambiente virtual de aprendizaje, cuyos OVAS integraron los juegos dentro de una historia denominada "El Enigma del Faraón".

Los preceptos teóricos giran en torno a las HSXXI y su situación en el contexto nacional e internacional. La solución de problemas en un contexto específico, como son los videojuegos tipo puzzle, también se profundiza en los AVA, los OVA y los juegos serios.

\section{Metodología}

Este estudio correspondió a una investigación correlacional en series cronológicas, con un solo grupo utilizando un diseño de tipo cuasi experimental.

La investigación se llevó a cabo durante el segundo semestre de 2011, con estudiantes de segundo semestre de la carrera de enfermería en la Fundación Universitaria de Ciencias de la Salud en Bogotá.

Se aplicaron tres pruebas diagnósticas. Posteriormente se interactuó con el AVA y se midieron los resultados en cada variable para luego ser correlacionados.

\section{Conclusiones}

Los resultados de las correlaciones realizadas entre las habilidades y la eficiencia como indicador en la solución de problemas, confirmaron que existe una influencia de estas sobre el desempeño en los juegos.

Existe una correlación entre la habilidad pensamiento sistémico y la solución de problemas: se pudo establecer que la correlación disminuía al estudiar la muestra por categorías.

Se encontró que los estudiantes con mayor tendencia al razonamiento deductivo reflejaron mejores resultados en la solución de problemas.

En la habilidad de trabajo colaborativo, los datos exponen que los estudiantes con dicha habilidad no presentaron mejores resultados en la solución de problemas. Aunque únicamente se halló un caso, el mismo podría confirmar la hipótesis principal de la investigación, en relación a que la existencia de estas habilidades en un individuo potencian su capacidad para dar solución a problemas tipo puzzle. 


\section{Título: Uso de categorías ontológicas en el aprendizaje colaborativo, mediante la solución de casos matemáticos utilizando mediaciones tecnológicas}

\section{Adriana Rocío Lizcano Dallos}

Palabras clave: Ontología, aprendizaje colaborativo, aprendizaje autónomo, resolución de problemas, ambiente de aprendizaje, argumentación y foros de discusión.

\section{Descripción}

Informe de trabajo de grado, cuyo propósito es identificar las diferencias en el aprendizaje colaborativo de grupos de estudiantes que resuelven problemas matemáticos orientados a la argumentación, que utilizan dos medios tecnológicos diferentes: 1) un sistema de argumentación que implementa categorías ontológicas (software Dígalo) y 2) un foro en línea.

\section{Contenidos}

El informe presenta los siguientes capítulos:

Aspectos preliminares: se hace una presentación del problema y los objetivos de investigación.

Metodología: se describe el diseño metodológico de la investigación, las características de la población que participó y las variables que hicieron parte del trabajo, así como los instrumentos de recolección de información, el procedimiento llevado a cabo y las técnicas de análisis de datos.

Antecedentes: en este capítulo se referencian las investigaciones en el área de aprendizaje colaborativo, evaluación del aprendizaje colaborativo, argumentación y software para la argumentación y uso de ontologías y argumentación, que proporcionaron conclusiones fundamentales en la formulación de este trabajo.

Marco teórico: se presentan los fundamentos teóricos que sustentan este trabajo, con el fin de proporcionar una visión más clara de las referencias, conceptos y metodologías, organizados en 6 ejes temáticos: aprendizaje autorregulado, aprendizaje colaborativo, ontologías, argumentación, software para la argumentación, y foros de discusión.

Ambiente de aprendizaje: se especifican los aspectos tenidos en cuenta para el diseño, desarrollo e implementación de los ambientes de aprendizaje con incorporación tecnológica que se utilizaron en la ejecución del trabajo, incluyendo el dominio de conocimiento, el modelo pedagógico y el modelo tecnológico.

Análisis e interpretación de datos: se presenta el análisis estadístico a través del software Statistical Package for the Social Sciences (SPSS) y la aplicación 
de la prueba T-Student, que permite determinar si existen diferencias significativas, y un análisis de contenido a los registros textuales generados en forma de mapas argumentativos, en el caso de Dígalo, y en forma de aportes en el caso de los foros de discusión, de acuerdo a los objetivos planteados en este trabajo investigativo. Finalmente, se hace la interpretación de resultados.

\section{Metodología}

La propuesta se enmarca dentro de la clasificación de investigación cuasi experimental, pues la asignación de grupos no es aleatoria, ya que se incorporan dentro del proceso dos grupos del curso de Matemáticas I de primer semestre de Ingeniería, de 25-35 estudiantes cada uno, preorganizados por la Universidad. De acuerdo con la clasificación planteada por Cook y Campbell (1979), corresponde a un diseño de grupo no equivalente sin pretest. Este tipo de diseño cuenta con dos grupos, uno experimental y otro de control no equivalente. La asignación no es aleatoria, lo que significa que no es posible garantizar la equivalencia de ambos grupos. Como su nombre indica, ninguno de los grupos ha tenido una medición previa a la introducción de la variable independiente. Así pues, el grupo experimental resolverá los problemas matemáticos utilizando un sistema de argumentación que implementa categorías ontológicas (software Dígalo) en la discusión, es decir, se somete al tratamiento, en tanto el grupo control resolverá los problemas matemáticos utilizando el foro de discusión, es decir, sin las categorías ontológicas.

\section{Conclusiones}

El análisis estadístico proporciona evidencias para afirmar que existen diferencias significativas, entre las características de aprendizaje colaborativo desarrolladas por los estudiantes que utilizan categorías ontológicas y los que utilizan foros en la solución de problemas matemáticos orientados a la argumentación. Esta diferencia se presenta a favor del uso de categorías ontológicas en las discusiones para la solución de problemas matemáticos.

Lo anterior permite validar la hipótesis alternativa que plantea que los estudiantes que realizan el proceso de discusión utilizando categorías ontológicas, obtienen un mejor desempeño en el trabajo colaborativo que los que utilizan foro de discusión, tanto en aspectos individuales como grupales.

Estos resultados son de relevancia para incentivar el uso de sistemas para la argumentación, que proporcionen categorías ontológicas y visualización gráfica de la discusión, en el marco del diseño de actividades cuyo objetivo es la solución de problemas matemáticos. Este planteamiento se encuentra alineado con los resultados de las investigaciones realizadas por Loll y Pinkwart (2011), que muestran que el uso de este tipo de sistemas mejora el trabajo colaborativo y la argumentación, y con las conclusiones de Scheuer, Loll, Pinkwart, y McLaren (2010) alrededor de las ventajas de la representación gráfica como una forma intuitiva de modelación de conocimiento, muy intuitiva y de uso común, que además facilita la expresión 
de las relaciones existentes entre los diferentes aportes de la discusión.

El análisis de contenido realizado a los registros obtenidos en Dígalo y los foros de discusión, muestra que el uso de categorías ontológicas en ambientes colaborativos para la solución de problemas matemáticos incentiva una mayor participación de los estudiantes en las discusiones: la utilización de expresiones que promueven el monitoreo de la solución y la formulación de estrategias para la orientación del trabajo del grupo.

Aunque los resultados obtenidos con el uso de categorías ontológicas a través de Dígalo, para incentivar el aprendizaje colaborativo, que arroja este trabajo son bastante positivos, es necesario considerar algunos aspectos negativos que se encuentran documentados en investigaciones previas y que igualmente fueron observados en esta experiencia. Dentro de estos aspectos se resalta la dificultad para la revisión de la secuencia de eventos en la discusión que presentan los mapas argumentativos obtenidos, al igual que la complejidad para la interpretación de los mismos por pares o evaluadores, hecho que ya habían documentado Scheuer, Loll, Pinkwart y McLaren (2010). Adicionalmente, la importancia de lograr que las categorías ontológicas sean lo suficientemente claras y diferenciadas (Scheuer, Loll, Pinkwart y McLaren, 2010), con un número adecuado de categorías (Loll y Pinkwart, 2011), de manera que para el estudiante sea más eficiente la identificación del tipo de aporte que desea hacer y por ende sea haga más pertinente su participación en la discusión.

La formulación de actividades de aprendizaje colaborativo que incorporan ambientes tecnológicos requiere de una clara definición de los objetivos (Collazos, Guerrero, Pino y Ochoa, 2002), una adecuada planeación, el aseguramiento de la funcionalidad del dispositivo tecnológico y un reconocimiento de la importancia de las intervenciones de los docentes en la discusión, pues esto se constituye en un factor clave en el proceso de enriquecimiento del trabajo de los estudiantes.

Adelantar procesos de medición del aprendizaje colaborativo requiere de una especificación cuidadosa de los instrumentos de valoración, que consideren tanto los aspectos individuales como grupales, que sean factibles de aplicar y con unos requerimientos de información apropiados, que permitan superar los inconvenientes planteados por Noble y Letsky, 2005. 